\title{
Idioms of Distress Among Trauma Survivors: Subtypes and Clinical Utility
}

\author{
Devon E. Hinton • Roberto Lewis-Fernández
}

Published online: 21 April 2010

(C) Springer Science+Business Media, LLC 2010

\begin{abstract}
In this introduction to the Special Issue on Trauma and Idioms of Distress, we provide an overview of the concept and typology of "idioms of distress," focusing particularly on their clinical utility. This includes the role of idioms as indicators of trauma exposure, of various types of psychopathology and of levels of distress, risk and functioning. It likewise includes the fact that idioms of distress may profoundly influence the personal meaning of having a trauma-related disorder, may shape the interpersonal course of the disorder and may pattern help-seeking and self-treatment. Finally, it illustrates the fact that idioms may also help clinicians understand sufferers' views of the causes of their distress, constitute key therapeutic targets and help increase therapeutic empathy and treatment adherence. This special issue focuses on the role played by idioms of distress in the local trauma ontology, the associations between the idioms and psychiatric disorders occurring in the context of trauma and the mechanisms by which the idioms profoundly influence the personal and interpersonal course of trauma-related disorders.
\end{abstract}

Keywords Trauma PTSD - Idioms of distress - Cultural syndromes · Clinical utility

\section{Introduction}

In this introduction to the Special Issue, we define what is meant by "idioms of distress," identify different subtypes of idioms of distress, and discuss various aspects of clinical utility afforded by identifying and assessing these idioms. By extension, we hope to illustrate how and why idioms of distress should be examined

D. E. Hinton $(\bowtie) \cdot$ R. Lewis-Fernández

Massachusetts General Hospital, 15 Parkman Street, WACC 812, Boston, MA 02114, USA

e-mail: devon_hinton@hms.harvard.edu 
in any adequate ethnography of trauma-related disorders. This taxonomy and discussion of clinical utility will provide an analytic framework to better situate the theoretical contributions to the broader literature of the articles in this special issue.

\section{Definition and Taxonomy of Idioms of Distress}

An idiom of distress was first defined by Mark Nichter (1981, p. 379) in the following way:

In any given culture, a variety of ways exist to express distress. Expressive modes are culturally constituted in the sense that they initiate particular types of interaction and are associated with culturally pervasive values, norms, generative themes, and health concerns.

Idioms of distress are those particular ways in which members of sociocultural groups convey affliction. Idioms vary across cultures, depending on the salient metaphors and popular traditions that pattern the human biological capacity for experiencing distress, including conditions that are sufficiently severe to meet criteria for psychiatric disorder. There are several types of idioms of distress (see Fig. 1). Some prototypical idioms include cultural illness syndromes (e.g., an ataque de nervios, literally, an "attack of nerves," among Caribbean-Latino populations or a khyâl attack among Cambodian refugees); a psychological or somatic complaint (e.g., being "sad," feeling bodily pain, being dizzy); religious involvement (e.g., being part of a possession cult); accusation of witchcraft assault (i.e., the assertion of having been attacked by means of sorcery or other supernatural mechanism); and acting-out behaviors (e.g., drinking). In each of these idioms,

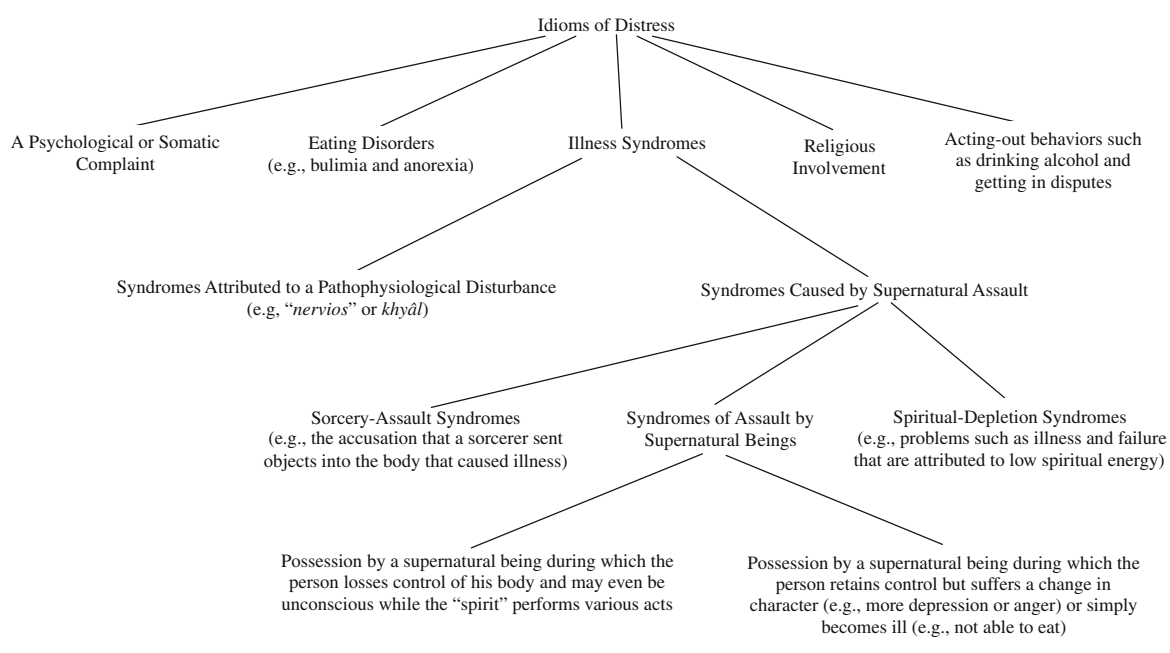

Fig. 1 The classification of idioms of distress 
culturally validated elements "run together" to form a relatively coherent whole, including folk etiologies, vulnerabilities, ethnopathological mechanisms, symptoms and expected remedies (Good 1977, 1994).

These patterns of responding to individually or collectively perceived distress are so embedded in everyday interactions that they are considered "common sense," sharing in a typically unquestioned normativity. For members of the culture, reality simply "is" this way. In their native setting, it is often difficult for observers to visualize the cultural relativity of these idioms, since they are just as "real" as anything else and thus unremarkable. To isolate them, investigators usually use examples from cultures other than their own, where precisely the "foreignness" of the idioms makes them stand out. The risk, of course, is to exoticize the topic itself, contributing to the idea that postindustrial Western societies are free of these expressions.

In an attempt to check this view, before proceeding to frame the clinical utility of idioms based on our work with Caribbean and Southeast Asian populations, we illustrate an idiom of distress with a Western example. This is the epidemic of "lower back pain," which is endorsed by as many as $26 \%$ of U.S. adults (Strine and Hootman 2007).

This U.S. idiom of distress is characterized by a web of cultural associations, which include overwork (as in the trope, "back-breaking labor"), general "stress", and even moral failure (e.g., "more than I can bear," "a monkey on his back," "spineless"). Other elements in the web of associations include precipitants such as insufficient interpersonal or job-related support or acute conflict in these areas. These associations link work-related and interpersonal tension specifically to the back muscles via a process of somatization, making them vulnerable to perceived pathology (Hinton and Good 2009; Kleinman et al. 1992). (In Latin America, by contrast, this kind of ethnopathological mechanism would link interpersonal stress preferably to somatized complaints based on local conceptions of the nervous system, resulting in the idioms of nervios (nerves) [Low 1985].) In addition, the somatic complaint of back pain is also associated with catastrophic cognitions, such as permanent anatomic harm and long-term disability, with limited expected improvement from biomedical treatments and with the use of alternative healing, such as chiropractic. At a societal level, anthropologists have connected the epidemic of lower back pain complaints to "the noxious effects of environmental hazards and power structures in the workplace, the crisis in the disability and welfare system, the negative consequences of medicalization, and the growth of a 'market' for pain products, pain professionals, and pain institutes" (Kleinman et al. 1992, p. 7).

As the example of back pain illustrates, idioms of distress constitute individual embodied expressions that are linked metaphorically to key conflicts at the interpersonal and societal level and that are often based on local ideas about the functioning of the body and mind. Physiologically, however, the conditions to which the idioms give rise are no less real as a result. Biological mechanisms are recruited for their expression and may result in chronic disease over time, especially in genetically or temperamentally vulnerable individuals. Back pain that results in inactivity, bad posture and excessive bed rest may yet develop into chronic muscle 
pathology (Koleck et al. 2006). Through this mechanism, behaviors and interpretations that originate as meaning-related expressions become embodied, and what starts out as an idiom of distress may end up also being a "disease," a biologically encoded pathology with its own self-perpetuating pattern (Kleinman et al. 1978).

Before moving on to the clinical utility of idioms of distress, it is useful to describe another key element of their typology: their time course. Some idioms correspond to a near-continual process, a traitlike condition; others are episodic, that is, they occur suddenly and then end, minutes or hours later. When the idiom of distress is a continual condition, it is often thought to predispose to yet other idioms of distress, which can be of either the continual or the episodic variety.

As an example, Latino patients may respond to life difficulties with a trait idiom of distress known as padecer de los nervios (to suffer from nerves), or having "altered nerves" (los nervios alterados), characterized by chronic anxiety, depression, somatization and/or dissociation. This condition is thought to result from the interaction of adversity and inherent vulnerability, an indigenous stressdiathesis model. The target organ of this vulnerability is the nervous systemparticularly the anatomical nerves themselves. A person with nervios is considered to be predisposed to recurrent emotional upset and anxiety and to development of other idioms of distress. If untreated and exacerbated by further adversity, for instance, chronic nervios is feared to lead to locura (madness), thought to be a violent and potentially incurable form of insanity characterized by delirium, indiscriminate violence and self-harm. This traitlike condition may be distinguished from another common consequence of chronic nervios: an episodic condition called ataques de nervios. These are acute crises that may involve screaming, crying, throwing things and experiencing severe somatic symptoms, such as trembling, palpitations and shortness of breath, and dissociative states, such as depersonalization-a syndrome most often found among Spanish speakers of the Caribbean (Guarnaccia et al. 2003).

As another example of the contrast of traitlike and episodic idioms of distress, a Cambodian refugee patient with certain symptoms-especially, frequent palpitations, startle and poor appetite and sleep-may consider him- or herself to be "weak" (khsaoy), to have a "weak heart" (khsaoy beh doung). Weakness, a chronic condition, is thought to predispose to episodic palpitations upon hearing loud noises (and possibly even cardiac arrest) and to having "khyâl attacks" (Hinton et al. 2002). In contrast, khyâl attacks are often the local label for acute anxiety and panic. Khyâl attack episodes last a relatively short period of time, minutes to hours, during which the person has many catastrophic cognitions about imminent bodily dysfunction-that khyâl and blood do not flow along the limbs, perhaps causing loss of the use of the arms and legs, and that khyâl and blood will surge upward in the body, possibly causing various physical complications.

\section{Clinical Utility of Idioms of Distress}

Idioms of distress matter, whether the goal is to provide culturally sensitive care or to conduct an adequate ethnography of trauma-related disorders in a certain cultural context. For example, if a clinician does not recognize complaints as culture-specific 
idioms (i.e., as opening up a complex network of meanings or, in other words, as revealing a hypersemiotized domain that links a complaint to multiple ontological zones), he or she may not attain a level of understanding of the patient's lifeworld critical to valid diagnostic practice and consensual treatment negotiation. Below we outline several aspects of clinical utility afforded by examination of a sufferer's idioms of distress.

\section{Indicators of Psychopathology}

Idioms of distress may be markers or expressions of psychopathology. This includes psychopathology dimensions (e.g., anxiety, depression or dissociation tendency) or specific disorders (e.g., PTSD and generalized anxiety disorder). These relationships can be investigated in studies that first separately assess idiom expression and the psychopathological constructs and then explore their relationship through analyses of association, such as odds ratios and correlation coefficients. Idioms of distress have been found to have consistently strong correlations with various psychiatric disorders, not a one-to-one relationship with one DSM disorder. Ataque de nervios, for example, is associated not just with panic disorder, but also with major depression, dissociative disorder NOS and several other DSM categories. This suggests that a "comorbidity approach" is a more fruitful way to study the relationship between idioms and diagnoses than prematurely subsuming the two (Lewis-Fernández et al. 2009a). This Special Issue examines how, in several cases, idioms of distress are associated with the presence of PTSD. In other cases, when a trauma survivor reports an idiom of distress, this may indicate that not only PTSD but also other comorbidities are likely to be present, such as the link among khyâl attacks and panic attacks and panic disorder.

\section{Indicators of Exposure to Trauma}

Whether certain idioms of distress are indicators of traumatic exposure has not been sufficiently studied. As examined in some of the articles in this issue, it is possible that in certain cultural contexts an idiom of distress may be a more sensitive indicator of traumatization than the diagnosis of PTSD. For instance, a cultural syndrome may be present when a person has suffered marked trauma and disability but does not fully meet PTSD criteria. In theory, the severity of the cultural syndrome may be more highly correlated with the total number of trauma events than PTSD severity.

\section{Indicators of Risk for Destructive Behaviors}

Idioms of distress are in some cases highly associated with destructive behaviors, such as substance abuse, episodes of violent anger or suicide attempts. For example, episodes of ataques may involve strong anger and its violent expression. Moreover, there is a strong relationship between ataque de nervios and suicidal ideation (odds ratio $[\mathrm{OR}]=6.2)$ and attempts $(\mathrm{OR}=8.1)$ (Guarnaccia et al. 1993). Multivariate regression with a national Latino community sample in the United States has 
confirmed this specific association. After adjusting for sociodemographic characteristics, lifetime ataque de nervios and traumatic exposure were each independently associated with suicidality, whereas a lifetime diagnosis of anxiety, depression or substance use disorder was not (Lewis-Fernández et al. 2009b).

\section{Indicators of Life Distress}

An idiom of distress may be a strong indicator of the general level of life distress experienced by an individual. Life distress can be of several types: interpersonal distress; health-related concerns about one's health or that of a loved one; concerns about personal safety, such as fear of physical or sexual assault in the living environment; and financial distress. Let us take the example of interpersonal distress.

Having a cultural syndrome may indicate that a person has interpersonal problems, such as spousal abuse, children who are acting out or conflicts with siblings. In many cultures, interpersonal conflict is considered to be a well-defined cause of certain cultural syndromes. Among Latino patients, for example, an episode of ataque de nervios often indicates a dispute involving the family (Guarnaccia et al. 1993), and so too, among Cambodian speakers, does a khyâl attack. In a Puerto Rican community sample, $92 \%$ of first ataque experiences were directly provoked by a distressing situation; $81 \%$ occurred in the presence of others and $67 \%$ led to the person receiving help. Contrary to the usual panic disorder, most respondents reported feeling better $(71 \%)$ or feeling relieved (81\%) after their first ataque. These findings suggest that first episodes of ataque de nervios are closely tied to the interpersonal world of the sufferer and that they result in an unburdening (desahogarse) of one's life problems, at least temporarily (Lewis-Fernández et al. 2005).

In many cultures, cultural syndromes play a key role as indicators of distress because of the salience attributed to worry as a factor in the syndrome's generation. The various zones of life distress often constitute "worry domains," also referred to as "worry topics." In many cultural syndromes across various cultural groups, worry episodes are often considered the cause of a continuous-type syndrome, predisposing to an episodic syndrome. Worry may also trigger events of the episodic syndrome: escalating worries about finances or troubled offspring, for example, a son who is in jail, may cause a predisposed Dominican patient to have an ataque de nervios or a Cambodian patient to have a khyâl attack. Through these mechanisms, worry about life-distress issues is linked to cultural syndromes.

\section{Indicators of Psychosocial Functioning}

Certain idioms of distress may indicate impairment in psychosocial functioning, including work and social functioning and general well-being, such as assessed by quality of life and disability measures: the complaint of "weakness" may be particularly associated with poor psychosocial functioning in Asian populations (Hinton et al. 2007). Likewise, U.S. Latinos with ataque de nervios had 2.3 times higher odds of being disabled due to a mental health problem than Latinos without 
ataque, after adjusting for sociodemographic and clinical characteristics, including the presence of depressive, anxiety and substance use disorders (Lewis-Fernández et al. 2009b). In traumatized groups, certain idioms of distress may significantly explain the variance in social functioning beyond that explained by PTSD severity: ataque status remained independently associated with mental health-related disability after adjusting for traumatic exposure and the presence of anxiety disorders, including PTSD (Lewis-Fernández et al. 2009b).

\section{Indicators of the Personal Meaning and Impact of Trauma-Related Disorders}

If a patient with a professionally diagnosed trauma-related disorder reports an idiom of distress, this will provide very useful information for the clinician. Experiencing the trauma and the trauma-related symptoms as an idiom of distress will profoundly influence the meaning and course of the trauma disorder for the person. It will create a certain identity: if a person identifies his or her distress as resulting from "PTSD" (if the patient attributes disorder to "PTSD," one must determine his or her conceptualization of the disorder), traumatic brain injury (TBI) or multiple chemical sensitivity, these each create a certain conception of the self, a certain life narrative: the idiom of distress becomes a part of the life narrative, of self-presentation. Additionally, the patient will be troubled by some culturally patterned concerns and not by others, such as the fear that the symptoms indicate a serious physical disorder or a distressing form of spirit possession. Moreover, depending on the idiom of distress, others in his or her social network will react to the person in certain ways: they may stigmatize the sufferer in certain ways - the person may self-stigmatize as well.

\section{An Indicator of the Help-Seeking Course for Trauma-Related Disorders}

If a patient attributes trauma-related symptoms to a certain idiom of distress (e.g., a particular cultural syndrome or a certain type of spirit possession), this will profoundly influence that patient's seeking out of treatment and the treatments used.

\section{Causes of Psychopathology}

Idioms of distress may play a key role in perpetuating various forms of psychopathology. Ataque de nervios, for example, is associated with elevated fears of anxiety symptoms that predispose the person to having panic attacks and panic disorder ("anxiety sensitivity" [Hinton et al. 2008a]). One possible explanation is that the ataque is related to a person's difficulty tolerating anxiety and other strong emotions (e.g., anger). In this view, the anxiety sensitivity is primary and derives from a relative intolerance of arousal because of the fear that arousal itself will lead to dangerous consequences (e.g., loss of control, violence). The ataque would then arise as a secondary reaction to the fear of arousal, as a sign that the person is overwhelmed by this fear and unable to cope in other ways. Alternatively-and this is the mechanism that concerns us now-it has been hypothesized that the idiom of distress itself may help elevate anxiety sensitivity. According to this alternative, 
arousal symptoms (e.g., racing thoughts, shortness of breath, shakiness of the limbs) are frightening precisely because of the concern that they will precipitate an ataque, which may then lead to a series of bodily catastrophes-such as losing control, going insane, being unable to breathe or having a seizure. Here the idiom acts as a primary precipitant of distress, as the source of catastrophic cognitions that cause panic attacks associated with the syndrome (Hinton and Good 2009). In other words, cultural constructs such as ataque may elevate anxiety sensitivity and cause panic attacks by providing a widely accepted ethnopathological mechanism linking the feeling of anxiety to specific bodily catastrophes (Hinton et al. 2008a). It has similarly been argued that catastrophic cognitions about anxiety symptoms generated by Cambodian cultural syndromes may result in high levels of anxiety sensitivity and frequent panic attacks (Hinton et al. 2006).

\section{Key Targets for Therapeutic Intervention}

In a cultural group, certain idioms of distress may play a key role in perpetuating distress and can be specifically targeted in treatment. The targeted idiom of distress may vary depending on the treatment aim: if the aim is to treat symptoms of GAD, panic or PTSD, the treatment should target those co-occurring cultural syndromes that generate fear about these types of symptoms and, in particular should address syndrome-associated catastrophic cognitions about somatic and mental symptoms. Illustrating this approach, in a CBT treatment of Cambodian refugees that targeted both PTSD and orthostatic panic-associated with a cultural syndrome, khyâl overload-improvement in PTSD was significantly mediated by improvement in orthostatic panic severity (Hinton et al. 2008b). In syndromes in which worry plays a key role, treatment should specifically focus on the worry topics that are signaled by the cultural syndromes, since these represent "experience-near" entry points for the intervention with clear face validity for the patient. In ataque de nervios, the immediate precipitant-for example, spousal disagreement or conflicts with the person's children triggered by acculturation differences-should be the first focus of psychotherapy.

\section{Facilitators of Empathy and Treatment Adherence}

In many groups, the distressed patient may consider an idiom of distress specific to that culture to be his or her most salient problem. If the clinician does not ask about this complaint and does not specifically address the syndrome-related concerns, the patient will likely not feel understood, thereby limiting therapeutic rapport and efficacy. Likewise, if the clinician indicates to the patient that the treatment (pharmacological or psychotherapeutic) will help relieve the idiom of distress, and ideally relieve the cause of distress as well as increasing the ability to handle distress, then this should greatly improve treatment adherence and, possibly, therapeutic success - if nothing else, by positive expectation and a placebo response. 


\section{Conclusion}

In this introduction to the Special Issue, we have delineated various types of idioms of distress and outlined their clinical utility to illustrate the advantages for a clinician of assessing for the presence of idioms of distress in a traumatized population. These same aspects of clinical utility constitute useful optics to guide research on idioms of distress. The articles in this Special Issue examine various types of idioms-particularly cultural syndromes and spirit possession-and illustrate their clinical utility. In particular, these papers focus on the role played by idioms of distress in the local trauma ontology, the associations between the idioms and psychiatric disorders occurring in the context of interpersonal trauma and the mechanisms by which the idioms profoundly influence the personal and interpersonal course of trauma-related disorders. Trauma does not occur in a vacuum: it develops and is understood in a particular cultural matrix, a key component of which are the idioms of distress. These constitute an essential component of the reticulum that connects the trauma event to the sufferer's body and to his or her social context.

\section{References}

Good, B.J.

1977 The Heart of What's the Matter: The Semantics of Illness in Iran. Culture, Medicine, and Psychiatry 1: 25-58.

1994 Medicine, Rationality, and Experience: An Anthropological Perspective. Cambridge: Cambridge University Press.

Guarnaccia, P.J., G. Canino, M. Rubio-Stipec, and M. Bravo

1993 The Prevalence of Ataques de Nervios in the Puerto Rico Disaster Study. Journal of Nervous and Mental Disease 181: 157-165.

Guarnaccia, P.J., R. Lewis-Fernández, and M. Rivera Marano

2003 Toward a Puerto Rican Popular Nosology: Nervios and Ataque de Nervios. Culture, Medicine, and Psychiatry 27: 339-366.

Hinton, D., and B. Good

2009 A Medical Anthropology of Panic Sensations: Ten Analytic Perspectives. In Culture and Panic Disorder. D. Hinton and B. Good, eds., pp. 57-84. Stanford: Stanford University Press.

Hinton, D.E., S. Hinton, K. Um, A. Chea, and S. Sak

2002 The Khmer "Weak Heart" Syndrome: Fear of Death from Palpitations. Transcultural Psychiatry 39: 323-344.

Hinton, D.E., V. Pich, S.A. Safren, M.H. Pollack, and R.J. McNally

2006 Anxiety Sensitivity among Cambodian Refugees with Panic Disorder: A Factor Analytic Investigation. Journal of Anxiety Disorders 20: 281-295.

Hinton, D.E., J. Sinclair, R.C. Chung, and M.H. Pollack

2007 The SF-36 among Cambodian and Vietnamese Refugees: An Examination of Psychometric Properties. Journal of Psychopathology and Behavioral Assessment 29: 38-45.

Hinton, D.E., R. Chong, M.H. Pollack, D.H. Barlow, and R.J. McNally

2008a Ataque de Nervios: Relationship to Anxiety Sensitivity and Dissociation Predisposition. Depression and Anxiety 25: 489-495.

Hinton, D.E., S.G. Hofmann, R.K. Pitman, M.H. Pollack, and D.H. Barlow

2008b The Panic Attack-PTSD Model: Applicability to Orthostatic Panic among Cambodian Refugee. Cognitive Behaviour Therapy 27: 101-116. 
Kleinman, A., L. Eisenberg, and B.J. Good

1978 Culture, Illness, and Care: Clinical Lessons from Anthropologic and Cross-Cultural Research. Annals of Internal Medicine 88: 251-258.

Kleinman, A., P.E. Brodwin, B.J. Good, and M.J. DelVecchio Good

1992 Pain as Human Experience: An Introduction. In Pain as Human Experience: An Anthropological Perspective. M.J. DelVecchio Good, P.E. Brodwin, B.J. Good, and A. Kleinman, eds., pp. 1-28. Berkeley: University of California Press.

Koleck, M., J.M. Mazaux, N. Rascle, and M. Bruchon-Schweitzer

2006 Psycho-Social Factors and Coping Strategies as Predictors of Chronic Evolution and Quality of Life in Patients with Low Back Pain: A Prospective Study. European Journal of Pain 10: 1-11.

Lewis-Fernández, R., P.J. Guarnaccia, S. Patel, D. Lizardi, and N. Díaz

2005 Ataque de Nervios: Anthropological, Epidemiological, and Clinical Dimensions of a Cultural Syndrome. In Perspectives in Cross-Cultural Psychiatry. A.M. Georgiopoulos and J.F. Rosenbaum, eds. Philadelphia: Lippincott, Williams, and Wilkins.

Lewis-Fernández, R., P.J. Guarnaccia, and P. Ruiz

2009a Culture Bound-Syndromes. In Kaplan and Sadock's Comprehensive Textbook of Psychiatry. B.J. Sadock, V.A. Sadock, and P. Ruiz, eds. Philadelphia: Lippincott, Williams, and Wilkins.

Lewis-Fernández, R., M. Horvitz-Lennon, C. Blanco, M. Alegría, and P.J. Guarnaccia

2009b Significance of Endorsement of Psychotic Symptoms by U. S. Latinos. Journal of Nervous and Mental Disease 197: 337-347.

Low, S.M.

1985 Culturally Interpreted Symptoms or Culture-Bound Syndromes: A Cross-Cultural Review of Nerves. Social Science and Medicine 21: 187-196.

Nichter, M.

1981 Idioms of Distress: Alternatives in the Expression of Psychosocial Distress: A Case from South India. Culture, Medicine, and Psychiatry 5: 379-408.

Strine, T.W., and J.W. Hootman

2007 U.S. National Prevalence and Correlates of Low Back Pain and Neck Pain among Adults. Arthritis and Rheumatism 57: 656-665. 